\title{
On the reliability of a PCA-based method for structural diagnosis in bridge structures with environmental disturbances
}

\author{
G. Quaranta ${ }^{1}$, B. Carboni ${ }^{2}$, and W. Lacarbonara ${ }^{2}$ \\ 1 University of California, Davis, CA 95616, USA \\ 2 Sapienza University of Rome. Via Eudossiana 18, 00184 Rome, Italy
}

\begin{abstract}
The basis for a vibration-based damage detection method is that changes in the constitutive properties of a structure will, in turn, modify its dynamic characteristics. Civil engineering structures are often subjected to time-varying environmental conditions and, as a consequence, the associated deviation of the identified structural features from the undamaged state may smear the changes caused by structural damages, thus causing non-issued alarms or false damage diagnoses. In order to assess their reliability for future in field applications, damage detection methods can be preliminarily validated by means of mechanical models in which the major changing environmental conditions are appropriately simulated. In doing so, two issues must be considered: the first is about the adopted thermomechanical model, the second deals with modeling realistic damage scenarios. To date, diagnostic tools are usually verified by means of oversimplified thermomechanical models, and the reliability of damage detection methods is demonstrated with reference to the damage severity only, whereas insufficient efforts have been directed toward the assessment of their effectiveness for the identification of spaceand time-varying damage scenarios. Through a refined three-dimensional thermomechanical model and a principal component analysis (PCA) diagnostic tool, this study addresses the feasibility of this specific class of damage detection methods for health monitoring of reinforced concrete bridge structures subject to realistic temperature effects.
\end{abstract}

\section{Introduction}

Vibration-based methods for structural diagnosis are powerful techniques for assessing the health of mechanical systems from dynamic measurements. However, their application in civil structures is still a challenging problem, because the system features are strongly affected by environmental factors other than damages. Field tests often reveal that the deviation of the system features caused by changes in environmental factors is significant, and can be larger than that due to important structural damages.

Several methods were proposed to alleviate environmental disturbances in vibration-based structural damage detection, i.e. artificial neural network [1], factor analysis [2], robust singular-value decomposition [3], principal component analysis (PCA) [4,5], support vector machine [6], missing data analysis [7], subspace-based approaches [8]. These non-parametric methods deal with the structural features only, since they do not require measurements of the environmental variables.

On the contrary, parametric methods require the calibration of prediction models to account explicitly for the effects of environmental factors [9]. Non-parametric diagnostic methods have some advantages over the parametric methods [4]. Within the latter, the environmental variables that affect the structural features must be defined in advance. Furthermore, once the correlation between the environmental variables and the measured features has been established, the failure of any of the sensors that measure the environmental variables may cause problems in the structural damage detection process. Numerical studies on the effectiveness of damage detection methods for structural diagnosis under varying environmental conditions are typ- ically based on simple structural models in which the coupled thermomechanical problem is addressed in a fairly approximate way $[4,10,11]$.

The most typical way for modeling damage scenarios via finite element models is based on the instantaneous reduction of the stiffness in some elements [4,7,10-13]. At the same time point-in-time damage scenarios are typically considered in laboratory applications [7]. Because of various practical and budget constraints, dynamic testing of real civil structures is also performed by introducing damages instantaneously [14] or on the time scale of a few days [15]. However, in most large civil structures damages appear as a relatively slow process whose spatial extension may expand in time. For instance, consider reinforced concrete $(\mathrm{RC})$ bridge structures subjected to pitting corrosion due to chlorides. Under this scenario, some zones of the bridge show time-varying stiffness reductions due to both cover cracking and steel corrosion. Moreover, since a deteriorating $\mathrm{RC}$ structure has the ability of redistributing the stress spatially, the damage due to pitting corrosion may expand because the chloride ions penetration process in concrete also depends on the current stress field [16]. As another example, one may consider the piers settlement, which is a typical slow damage scenario for some bridge structures. Existing damage detection methods for structural diagnosis under varying environmental conditions were essentially tested with reference to instantaneous damage scenarios, thus assessing their reliability with respect to the damage severity only. None of the existing studies addressed appropriately their effectiveness with respect to more realistic space- and time-varying damage scenarios. Nonetheless, deterioration rates as well as de- 
terioration patterns are likely to affect seriously the reliability of vibration-based methods for structural diagnosis. Within this framework, the present work discusses the reliability of structural diagnostic methods for reinforced concrete (RC) bridge structures under environmental disturbances. To this end, the environmental conditions are simulated through an accurate three-dimensional (3D) continuum procedure [17]. Different damage scenarios - such as stiffness reductions and piers settlements - are examined. These scenarios are introduced in the model through instantaneously prescribed variations of some parameters as well as through time- and/or space-varying processes. A PCA-based diagnostic method is adopted for the purpose of structural diagnosis.

\section{Bridge structure}

\subsection{Modal analysis under thermal gradients}

In order to evaluate the temperature field in a $\mathrm{RC}$ bridge, a direct approach to the thermal problem is proposed by considering geographically referenced structures in their full tridimensionality. The structure is subjected to heat exchanges with the environment due to solar radiation, convection, and radiation. This scenario corresponds to the mixed boundary conditions of the Fourier-Robin type which are added to the general heat equation. Convection and radiation between the structure and the surrounding air fluid are defined through the measured trend of air temperature. For the solar radiation, the same approach employed for the design of photovoltaic panels is adopted. According to the geographical location, clearness index, day of the year, and geographical orientations of the surfaces, the methodology provides the three components of solar radiation.

The changes in frequencies are due to thermo-geometric stiffness and the variation of the concrete elastic constants with temperature. The thermal dependence of Young's modulus can be represented in the considered range of temperatures [18] by the following linear equation:

$$
E(\boldsymbol{x}, t)=E_{20^{\circ} \mathrm{C}} \cdot\left[1-\eta_{e}\left(\vartheta(\boldsymbol{x}, t)-20^{\circ} \mathrm{C}\right)\right]
$$

where $\vartheta(\boldsymbol{x}, t)$ is the temperature field at time $t$ in the material, $E_{20^{\circ} \mathrm{C}}$ is Young's modulus at $20^{\circ} \mathrm{C}$ and $\eta_{e}$ is the temperature coefficient of Young's modulus, which is suggested as $0.003\left[1 /{ }^{\circ} \mathrm{C}\right]$ by CEP/FIP recommendations [19].

The mechanical problem is governed by the nonlinear Cauchy equations of motion. Simulation of the described heat diffusion problem are conducted for a period of one year and the obtained temperature fields are the input data for the thermoelastic problem. Every four hours the thermoelastic equilibrium configuration is computed. The linearized eigenvalue problems are solved thus obtaining the frequencies of the structures.

\subsection{Three-dimensional model}

The structure is a RC bridge resting on steel-reinforced rubber supports. The boundary conditions are then assumed to provide a simply supported-like scheme. In particular, the areas spanned by the supports are fixed in the left end while the areas in the right end are subject to constraints only in vertical and lateral directions, thus leaving the horizontal displacement free. The deck is $59 \mathrm{~m}$ long and is made of concrete whose Young's modulus is $30.24 \mathrm{GPa}$ at the temperature of $20^{\circ} \mathrm{C}$, Poisson's ratio is 0.2 , and the mass density is $\rho=2500 \mathrm{~kg} / \mathrm{m}^{3}$. The thermal features assumed for the computations are: heat capacity $c=800$ $J /(K \cdot k g)$, conductivity coefficient $k=1.8\left(W \cdot K^{-1} \cdot m^{-1}\right)$, thermal exchange coefficient $\left(W \cdot m^{-2} \cdot K^{-1}\right)$, and $\epsilon=\alpha=$ 0.85 are respectively the emissivity and the absorptivity coefficients.

The bridge is located in Rome (latitude $43^{\circ} 14^{\prime} 24^{\prime \prime} \mathrm{N}$ ) with its longitudinal axis collinear with the direction from North to South. The orientation and inclination of the outer surfaces of the bridge are accordingly defined to determine the boundary conditions for the heat diffusion problem. The deck section is closed at the supports while it is hollow along the span.

In Figs. 1 and 2 the mesh of the structure and the temperature field at 2:30 p.m. on July 21 are shown.

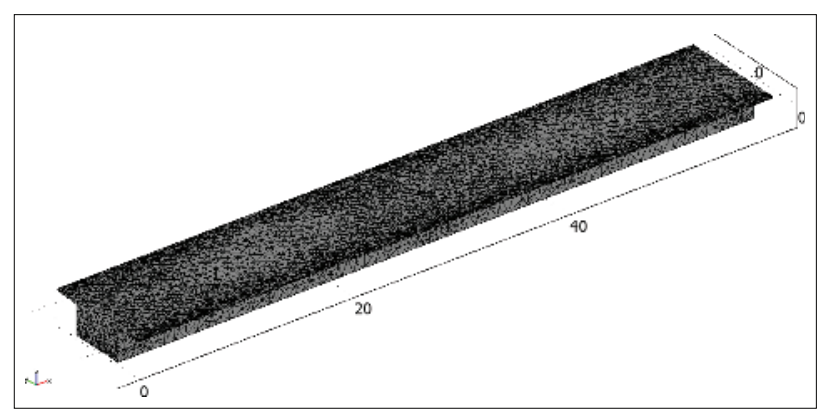

Fig. 1. Finite element mesh: 61733 tetrahedra.

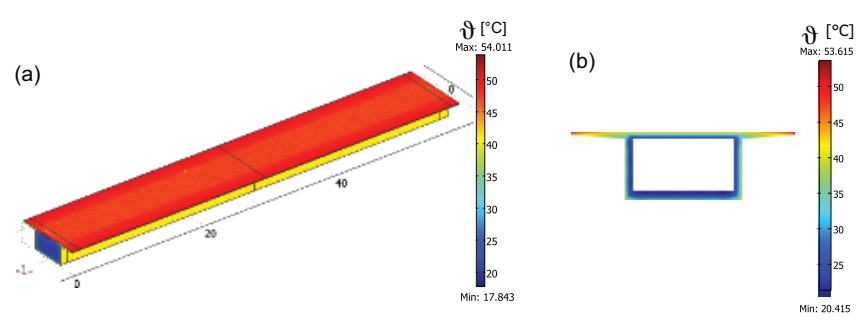

Fig. 2. Temperature distribution on July 21,2010 at 2:30 pm for a clearness index $K t=0.5$. (a) $3 \mathrm{D}$ temperature contour plot, (b) temperature plot in the midspan cross section.

\section{Damage modeling and identification}

There are different types of structural damage. In this work considered two types are : pitting corrosion and piers set- 
tlement. The first is modeled with a time- and/or spacevarying stiffness reductions in term of Young's modulus is some zones of the structure. The second is modeled prescribing displacements to the supports and computing the new equilibrium configurations and associated frequencies. In most large civil structures, damages appear as a relatively slow process whose spatial extension may expand in time, however existing damage detection methods were tested with reference to instantaneous damage scenarios. In order to assess the difference between these two different ways of considering the appearance of damage, we have considered eight different scenarios described in detail below.

\subsection{Damage scenarios}

The damage scenarios considered are labeled by letter D followed by a number:

- D101: at times 1095 and 1643, two instantaneous reductions of Young's modulus $(30 \%)$ are given in the whole section respectively at $1 / 2$ and $3 / 4$ of the span length for an extension of $25 \mathrm{~cm}$ in the longitudinal direction of the bridge;

- D102: at times 1095 and 1643, two instantaneous reductions of Young's modulus (30\%) are prescribed respectively at $1 / 2$ and $3 / 4$ of the span length for an extension of $25 \mathrm{~cm}$ in the longitudinal direction and 50 $\mathrm{cm}$ in the transversal direction of the bridge;

- D201: at times 1095, an instantaneous settlement of 12 $\mathrm{cm}$ of the two diagonally opposite supports is given;

- D202: at times 1095 an instantaneous settlement of 6 $\mathrm{cm}$ of the two diagonally opposite supports is prescribed;

- D301: from time 1095 to 1460 (duration 2 months), a spatial and temporal evolution of the damage in terms of reduction of Young's modulus occurs; at time 1460, this reduction is $30 \%$, and affects the entire midspan section for an extension of $25 \mathrm{~cm}$ in the longitudinal direction of the bridge; from time 1643 to 1825 (duration: 1 month) a spatial evolution of the damage takes place; at the end, the reduction of the modulus is $30 \%$, and occurs throughout the section at three-quarters of the span for an extension of $25 \mathrm{~cm}$ in the longitudinal direction of the bridge;

- D302: from time 1095 to 1825 (duration 4 months), a spatial and temporal evolution of the damage occurs in terms of reduction of Young's modulus; at time 1825, this reduction is $30 \%$ and occurs over the entire midsection for an extension of $25 \mathrm{~cm}$ in the longitudinal direction of the bridge;

- D4101: from time 1095 to 1460 (duration 2 months), a time-varying settlement of two diagonally opposite supports occurs; at time 1460, the settlement is $12 \mathrm{~cm}$;

- D402: from time 1095 to 1641 (duration 3 months), a time-varying settlement of two diagonally opposite supports; at time 1641, the settlement is $6 \mathrm{~cm}$; from time 1641 to 1823 (duration 1 month), an additional settlement takes place until reaching the value of $6 \mathrm{~cm}$.

\subsection{Linear PCA for structural diagnosis}

The linear PCA-based method proposed in Ref. [4] is employed to deal with structural diagnosis problems under environmental disturbances. The linear PCA proposed in Ref. [4] can be used when environmental conditions are such that they have a linear or weakly nonlinear effect on the vibration features. In this paper, system frequencies are selected as damage sensitive features of the bridge. As long as the modal analysis reveals that the linear assumption holds true for the frequencies of the examined RC bridge, the linear PCA can be applied effectively. Structural diagnostics by frequency changes attracted a lot of interest because instrumentation requirements are relatively low (few sensors can be sufficient), the procedure of extracting eigenfrequencies is relatively simple and high accuracy can be achieved using longer measurement periods. However, the modal analysis of the considered bridge structure confirmed that the sensitivity of the frequencies due to most damage cases of interest is low. At the same time, frequency changes are heavily smeared by environmental factors. Therefore, a critical concern deals with the reliability of a system frequencies-driven PCA to indicate the occurrence of damages.

The measured frequencies are collected in the samples matrix $\boldsymbol{Y} \in R^{n \times N}$ where $n$ is the number of frequencies and $N$ the number of samples. Extracting the main $m$ eigenvectors of the covariance matrix of $\boldsymbol{Y}$ defined as $\boldsymbol{R}=1 / N \boldsymbol{Y} \boldsymbol{Y}^{\mathrm{T}}$ ), the loading matrix $\boldsymbol{T}$ can be defined as the collection of eigenvectors through which the samples can be mapped from the original dimension $n$ to the lower dimension $m$ :

$$
X=T Y \text {. }
$$

Projecting the data back to the original dimension, the following reduced matrix is obtained:

$$
\widehat{\boldsymbol{Y}}=\boldsymbol{T}^{\mathrm{T}} \boldsymbol{X}=\boldsymbol{T}^{\mathrm{T}} \boldsymbol{T} \boldsymbol{Y}
$$

The loss of information is contained in the residual error matrix:

$$
E=Y-\widehat{Y}
$$

By considering the residual error vector $\boldsymbol{E}_{k}$ at time $t_{k}$, the novelty index is defined through the Mahalanobis norm based on the covariance matrix of $\boldsymbol{Y}$ :

$$
N I_{k}=\sqrt{\boldsymbol{E}_{k}^{\mathrm{T}} \boldsymbol{R}^{-1} \boldsymbol{E}_{k}^{\mathrm{T}}}
$$

\section{Numerical investigation}

Figure 3 shows the frequencies during the year for the undamaged bridge and the damage scenario D101. The range of frequency variation of the undamaged bridge is in agreement with the reference paper [17]. Figure 4 portrays the frequencies with respect to the lowest frequency for damage scenarios D101 and D102. A linear correlation is observed which justifies the use of linear PCA. Mahalanobisbased novelty indices by linear PCA are shown in Fig. 5. The method clearly detects the presence of point-in-time elastic modulus reductions when they occur with a rather large magnitude (D101) whereas less severe instantaneous damage scenarios are not identified (D102). The method is also capable of detecting time-dependent elastic modulus reductions (D301, D302). However, if the deterioration process is too slow, a delay occurs in identifying when it gets started, meaning that the occurrence of the damage can be identified only if the damage severity is large 
enough. This is evident in D302 and in the first damage event of D301. The spatial variation of the elastic modulus (second damage event of D301) is well detected. On the other hand, it appears that the method fails in identifying damage scenarios associated with the bridge pier settlements, since point-in-time events (D201, D202) as well as time-dependent phenomena (D401, D402) are not easily detected from Fig. 5

\section{Conclusion}

This work suggests the possibility of using an accurate $3 \mathrm{~d}$ thermomechanical model to obtain realistic damage scenarios in the presence of environmental disturbances on which the PCA structural diagnosis tool is tested assuming the modal frequencies as sensitive features. The results demonstrate the need to evaluate the space-time evolution of damage, first because the level of detected damage severity can be estimated only in this way and, second, because several damage types in civil structures appear as a relatively slow process whose spatial extension severity expands in time. Not all types of damage are detected such as the pier settlements which, for this kind of structure, do not influence significantly the frequencies. In conclusion, accurate predictive models for each individual structure must be considered before any modal-based damage identification algorithms can be applied with a certain degree of confidence.
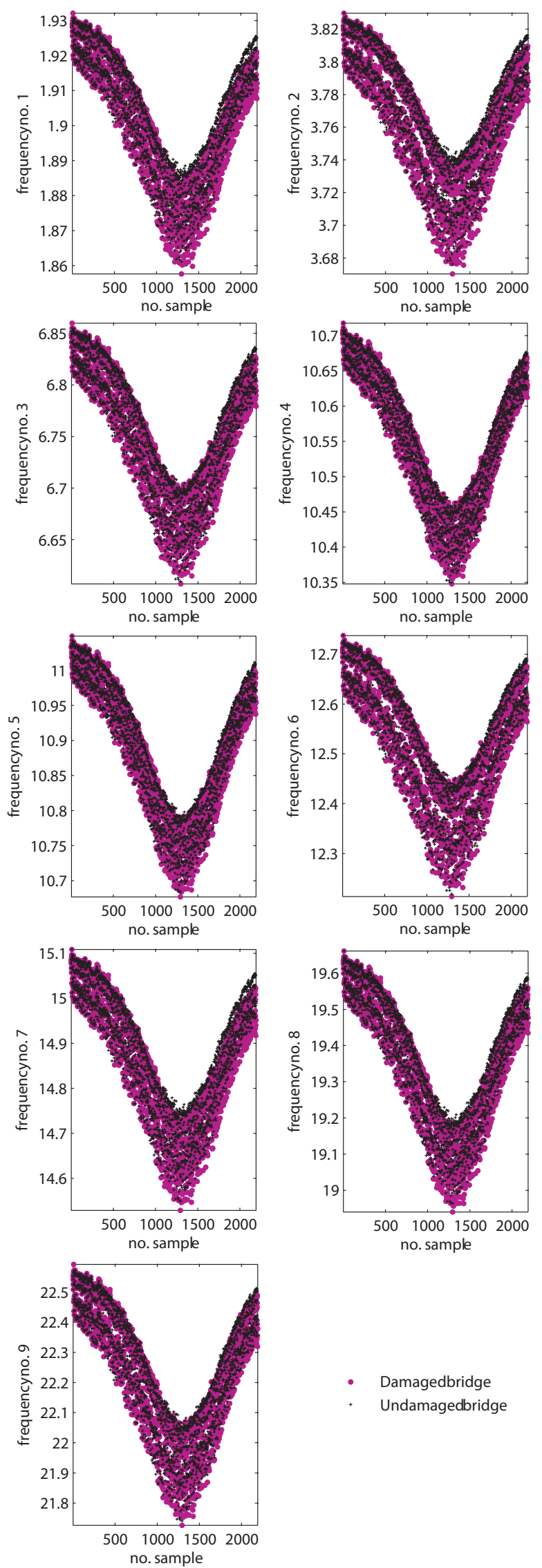

Damagedbridge Undamagedbridge

Fig. 3. Frequency variation for undamaged and damaged (damage scenario: D101) bridge structure. 

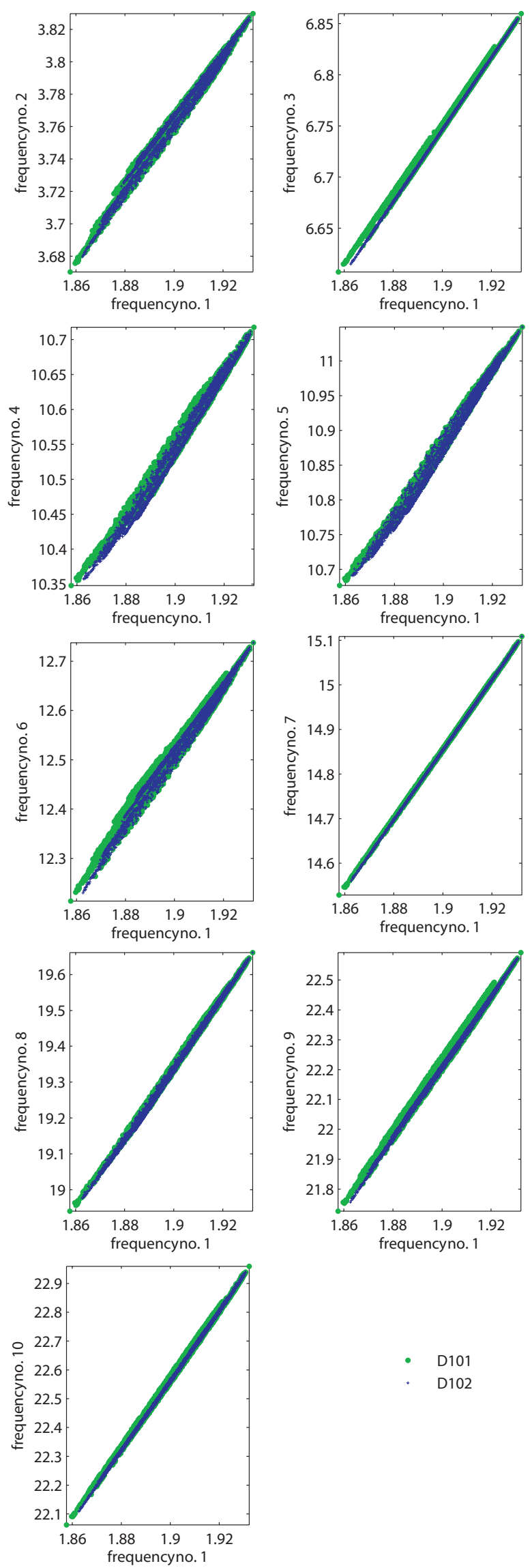

Fig. 4. Comparison between two damage scenarios.
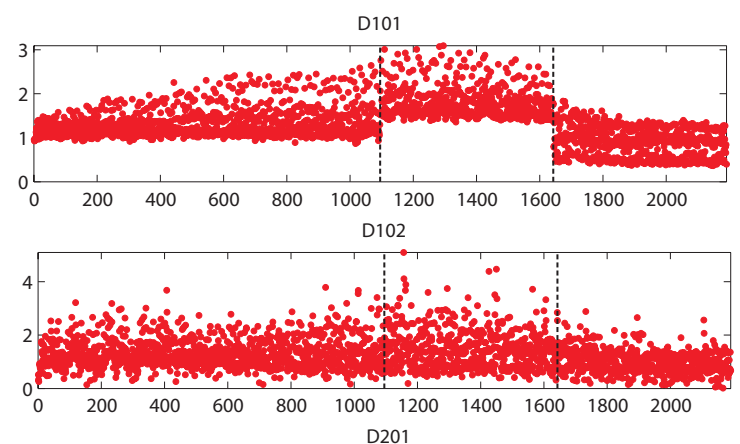
D201

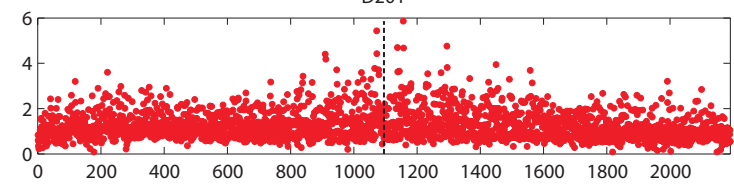
D202
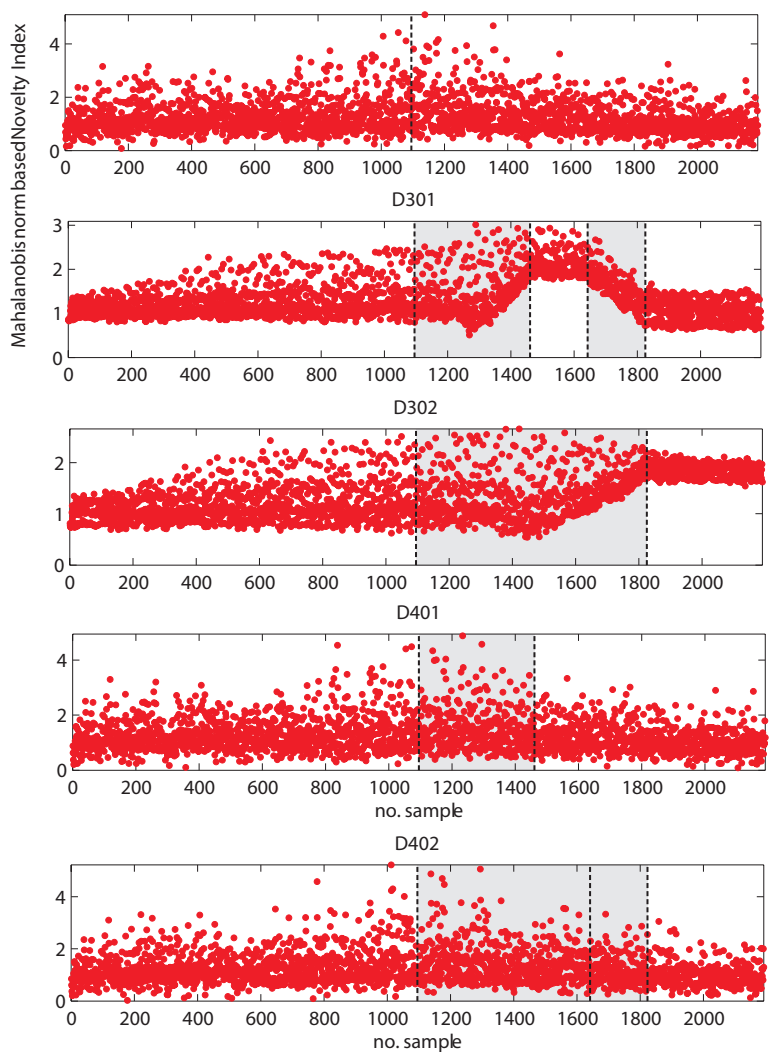

Fig. 5. Novelty indices for the examined damage scenarios. 


\section{References}

1. H. Sohn, K. Worden, C.R. Farrar, Statistical damage classification under changing environmental and operational conditions, J.Intel. Mat. Syst. Str. 13, 92002

2. Elimination of environmental influences from damagesensitive features in a structural health monitoring system, Proc. First European Workshop on Structural Health Monitoring, Lancaster, Pennsylvania (USA), 742-749, 2002.

3. S. Vanlanduit, E. Parloo, B. Cauberghe, P. Guillaume, P. Verboven,v3. S. Vanlanduit, E. Parloo, B. Cauberghe, P. Guillaume, P. Verboven, A robust singular value decomposition for damage detection under changing operating conditions and structural uncertainties, J. SoundVib., 284, 1033-1050, 2005.

4. A. M. Yan, G. Kerschen, P. De Boe, J. C. Golinval, Structural damage diagnosis under varying environmental conditions - part I: a linear analysis, Mech. Syst. Signal Pr. 19, 847-864, 2005.

5. A. M. Yan, G. Kerschen, P. De Boe, J. C. Golinval, Structural damage diagnosis under varying environmental conditions - part II: local PCA for non-linear cases, Mech. Syst. Signal Pr. 19, 865-880, 2005.

6. C. K. Oh, H. Sohn, Damage diagnosis under environmental and operational variations using unsupervised support vector machine, J. Sound Vib.,325, 224-239, 2009.

7. J. Kullaa, Eliminating environmental or operational influences in structural health monitoring using the missing data analysis, J. Intel. Mat. Syst. Str. 20, 1381-1390, 2009.

8. M. Basseville, F. Bourquin, L. Mevel, H. Nasser, F. Treyssède, Handling the temperature effect in vibration monitoring: two subspace-based analytical approaches, J. Eng. Mech.-ASCE,136, 367-378, 2010.

9. M. Ralbovsky, S. Deix, R. Flesch, Frequency changes in frequency-based damage identification, Struct. Infrastruct. E. 6, 611-619, 2010.

10. A. Deraemaeker, E. Reyndersb, G. De Roeck, J. Kullaa, Vibration-based structural health monitoring using output-only measurements under changing environment, Mech. Syst. Signal Pr. 22, 34-56, 2008.

11. T.-Y. Hsu, C.-H. Loh, Damage detection accommodating nonlinear environmental effects by nonlinear principal component analysis, Struct. Control Hlth. 17, 338354, 2010.

12. D. F. Giraldo, S. J. Dyke, J. M. Caicedo, Damage detection accommodating varying environmental conditions, Struct. Health Monit. Struct. Health Monit. 5, 155172, 2006.

13. D. Posenato, P. Kripakaran, D. Inaudi, I.F.C. Smith, Methodologies for model-free data interpretation of civil engineering structures, Comput. Struct. 88, 467-482, 2010.

14. M. Dilena, A. Morassi, Dynamic testing of a damaged bridge, Mech. Syst. Signal Pr. 25, 1485-1507, 2011.

15. C. Kramer, C. A. M. De Smet, G. De Roeck, Z24bridge damage detection tests, Proc. of IMAC 17, Kissimmee, Florida (USA), 1023-1029, 1999.

16. L. Guoping, H. Fangjian, W. Yongxian, Chloride ion penetration in stressed concrete, J. Mater. Civil Eng. 23, 1145-1153, 2011.
17. B. Carboni, W. Lacarbonara, A three-dimensional continuum approach to the thermoelastodynamics of large-scale structures. Eng. Struct. doi:10.1016/j.engstruct.2012.02.008.

18. D. J. Naus, Report of Oak Ridge National Laboratory, Washington, 2006.

19. CEB-FIP Model Code, London, Thomas Telford 1993. 